\title{
Modelling, screening, and solving of optimisation problems: Application to industrial metal forming processes
}

\author{
M.H.A. Bonte, A.H. van den Boogaard and E. Veldman \\ University of Twente, P.O. Box 217, 7500 AE Enschede, The Netherlands
}

\begin{abstract}
Coupling Finite Element (FEM) simulations to mathematical optimisation techniques provides a high potential to improve industrial metal forming processes. In order to optimise these processes, all kind of optimisation problems need to be mathematically modelled and subsequently solved using an appropriate optimisation algorithm. Although the modelling part greatly determines the final outcome of optimisation, the main focus in most publications until now was on the solving part of mathematical optimisation, i.e. algorithm development. Modelling is generally performed in an arbitrary way.

In this paper, we propose an optimisation strategy for metal forming processes using FEM. It consists of three stages: a structured methodology for modelling optimisation problems, screening for design variable reduction, and a generally applicable optimisation algorithm. The strategy is applied to solve manufacturing problems for an industrial deep drawing process.
\end{abstract}

Keywords: optimisation modelling, screening, optimisation algorithm, metal forming, FEM

\section{INTRODUCTION}

Product improvement and cost reduction have always been important goals in the metal forming industry. The rise of Finite Element simulations for metal forming processes has contributed to these goals in a major way. More recently, coupling FEM simulations to mathematical optimisation techniques has shown the potential to make a further giant contribution to product improvement and cost reduction.

Mathematical optimisation consists of the modelling and solving of optimisation problems. Much research on the optimisation of metal forming processes has been published during the last couple of years, see e.g. [1]. Most of this research focussed on the solving part of optimisation, i.e. the development of a specific algorithm and its application to a specific optimisation problem for a specific metal forming process. Although the modelling of the optimisation problem determines the final outcome of optimisation, it is often done quite arbitrarily. To our opinion, much more attention should be paid to the proper modelling of optimisation problems in metal forming, rather than implementing another slightly more efficient optimisation algorithm.

In this paper, we propose a generally applicable optimisation strategy which makes use of FEM simulations of metal forming processes. The strategy includes both a structured methodology for modelling and an algorithm for solving optimisation problems. The optimisation strategy is applied to the optimisation of an automotive deep drawing process.

CP907, $10^{\text {th }}$ ESAFORM Conference on Material Forming, edited by E. Cueto and F. Chinesta (C) 2007 American Institute of Physics 978-0-7354-0414-4/07/\$23.00 


\section{THE OPTIMISATION STRATEGY FOR METAL FORMING PROCESSES}

The proposed optimisation strategy consists of three stages: modelling, screening and solving.

\section{Modelling}

The first stage is to model the optimisation problem, i.e. defining objective function, constraints and design variables. We propose a structured 7 step methodology for modelling optimisation problems in metal forming [2]:

1. Determine the appropriate optimisation stage

2. Select only the necessary responses

3. Select one response as objective function, the others as implicit constraints

4. Quantify the objective function and implicit constraints

5. Select possible design variables

6. Define the ranges on the design variables

7. Identify explicit constraints

Being based on the generally applicable Product Development Cycle [2, 3], this modelling methodology can be applied to any metal forming process, product and problem. In the end, it yields a specific mathematical optimisation model, which can subsequently be solved using a suitable optimisation algorithm. The 7 step methodology is further demonstrated in the next section when it is applied to an industrial deep drawing process.

\section{Screening}

If many design variables are still present in the modelled optimisation problem, screening techniques can be applied to reduce the number of design variables. We propose to apply a Resolution III fractional factorial DOE strategy [5] for screening. Resolution III designs allow for independently estimating the linear effects of the design variables on the responses (objective function and constraints). After having run the corresponding FEM simulations, the linear effects can be estimated by applying statistical techniques such as ANalysis Of VAriance (ANOVA) [5]. The amount and direction of the effect of each variable on each response can be nicely displayed in Pareto and Effect plots. Examples of Pareto and Effect plots are presented in the Figures 1(a) and (b), respectively.

Using these techniques, the variables with the largest effects may be kept in the optimisation model whereas the variables having less effect may be omitted. In such a way, the amount of design variables may be significantly decreased while maintaining control over objective function and constraints during optimisation. 


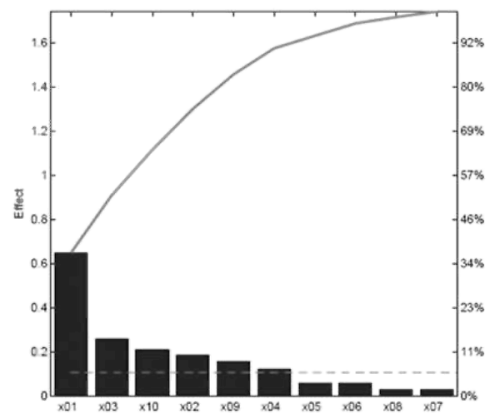

(a)

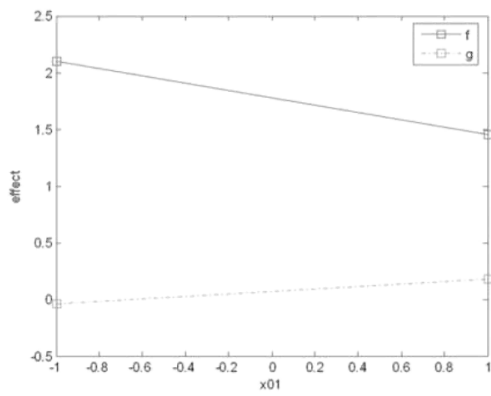

(b)

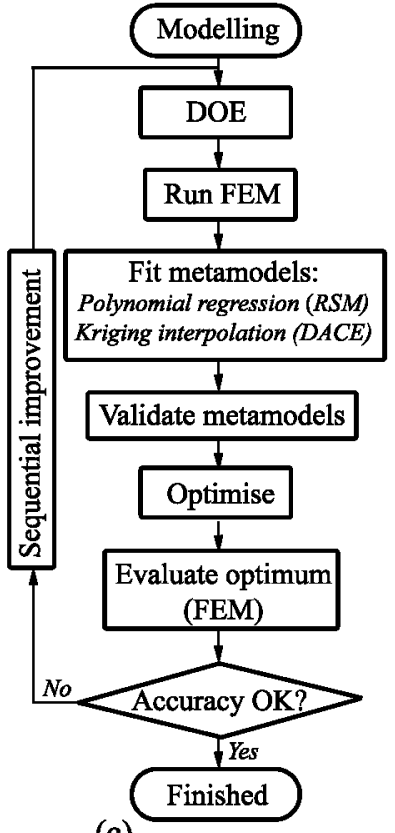

(c)

FIGURE 1. (a) Pareto plot; (b) Effect plot; (c) SAO [4]

\section{Solving}

The final stage of the optimisation strategy is to solve the optimisation problem by a suitable algorithm. We propose the Sequential Approximate Optimisation (SAO) algorithm presented in several publications, see e.g. [4]. An overview of the algorithm is presented in Figure 1. It comprises a spacefilling Latin Hypercubes Design Of Experiments (DOE) strategy, RSM and Kriging metamodelling and validation techniques, and a multistart SQP algorithm for optimising the metamodels. The algorithm allows for sequential improvement of the accuracy.

\section{APPLICATION TO DEEP DRAWING}

The optimisation strategy presented in the previous section is applied to an industrial metal forming process. The automotive part concerned is shown in Figure 2(a). It is manufactured from $3 \mathrm{~mm}$ thick steel sheet in 19 stages, of which 10 are deep drawing operations.

In an early stage, cracks were encountered during or after the deep drawing process ("reference process"). The location of the cracks is indicated in Figure 2(a). To reduce 


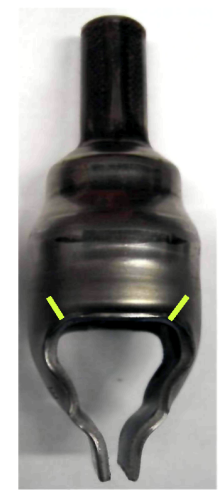

(a)

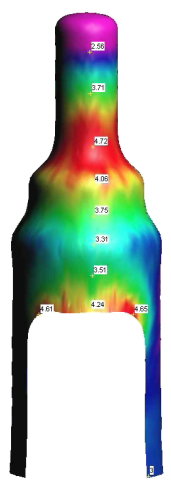

(b)

FIGURE 2. The automotive part: (a) Part and crack location; (b) FEM simulation

the scrap rate, some stages of the deep drawing process have been altered resulting in a significant decrease of the defect rate ("modified process").

To decrease material and control costs, the optimisation strategy proposed in the previous section is applied to this production problem. Two optimisation cases will be treated: (i) optimisation of the reference process; and (ii) optimisation of the modified process.

\section{Modelling}

Steps 1,2, 3 and 4 of the 7 step methodology for modelling the optimisation problem yielded the following response model:

$$
\begin{array}{r}
\min f=\frac{d_{f 1}}{d_{f 1 r e f}}+\frac{d_{f 2}}{d_{f 2 r e f}} \\
\text { s.t. } g=d_{g} \leq 0
\end{array}
$$

$f$ is the objective function that aims to reduce the crack occurrence. Two causes of the cracks have been determined [6]: (i) large deformations in the critical region; and (ii) a thickness concentration in the critical region. One can observe the thickness concentration in the FEM calculation presented in Figure 2(b). AutoForm has been adopted as FEM code. Both causes are visualised in the Forming Limit Diagram (FLD) in Figure 3(b): $d_{f 1}$ depicts the definition of the amount of deformation in the critical region, $d_{f 2}$ the distance to the line of equal thickness which represents the thickness in the critical region. $d_{f 1 \text { ref }}$ and $d_{f 2 r e f}$ are the objective function values corresponding to the reference process. As an implicit constraint, necking is not allowed to occur. $d_{g}$ is the distance to the material dependent Forming Limit Curve (FLC), which is a measure 


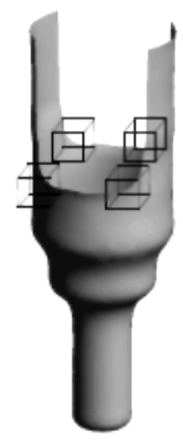

(a)

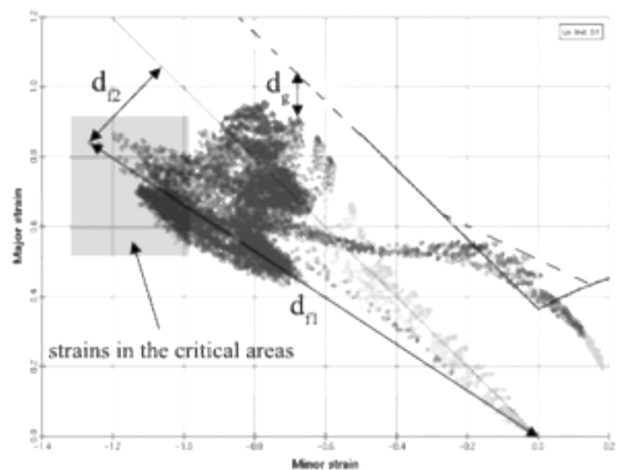

(b)

FIGURE 3. (a) Definition of critical region; (b) Definition of responses

for necking. This response is not bounded to the critical region: necking is not allowed at any location throughout the automotive part.

Steps 5,6 and 7 of the modelling methodology assisted in defining 10 design variables for this application: the blank size and thickness, punch and die radii and blank holder forces of several deep drawing operations, and coefficients of friction.

\section{Screening}

Ten design variables is, however, still quite many to optimise using the SAO algorithm. Therefore, a $2_{\text {III }}^{(10-6)}$ design has been generated and the corresponding 16 FEM calculations have been conducted. ANOVA has been applied and the Pareto and Effect plots of $f$ and $g$ have been generated: Figure 1(a) showed the Pareto plot of $f$, Effect plots of $f$ and $g$ were displayed in Figure 1(b). It can be seen that the blank size is the most dominant variable. The influence of the other variables has been attributed to numerical noise. Hence, the blank size was the only variable taken into account for optimisation. The other 9 variables were kept at their original settings.

\section{Solving}

The one variable optimisation problem has subsequently been solved using SAO. Table 1 presents the results, which were obtained after running 14 FEM calculations. Applying the optimisation strategy significantly reduced the objective function value of the reference process. The modified process still performs somewhat better. Table 1 also presents the results of optimising the modified process [6]. Note the further, significant improvement of the objective function, which suggests cracks can be reduced further using the proposed optimisation strategy. 
TABLE 1. Optimisation results

\begin{tabular}{lrrrr}
\hline & $\begin{array}{r}\text { Reference } \\
\text { process }\end{array}$ & $\begin{array}{r}\text { Modified } \\
\text { process }\end{array}$ & $\begin{array}{r}\text { Optimised } \\
\text { reference } \\
\text { process }\end{array}$ & $\begin{array}{r}\text { Optimised } \\
\text { modified } \\
\text { process }\end{array}$ \\
\hline Blank size & 204 & 204 & 209.9 & - \\
$\mathrm{f}$ & 2 & 1.4823 & 1.6732 & 0.9097 \\
$\mathrm{~g}$ & -0.0171 & -0.0459 & -0.0049 & -0.0048 \\
\hline
\end{tabular}

\section{CONCLUSIONS}

A generally applicable optimisation strategy for metal forming processes using FEM has been proposed. It contains a structured methodology for modelling optimisation problems, screening techniques for design variable reduction, and a generally applicable algorithm for solving the optimisation problem. The strategy has been successfully applied to an industrial deep drawing process. The strategy has been and is being applied to other industrial metal forming processes, products and optimisation problems as well, which indicates its general applicability.

\section{ACKNOWLEDGMENTS}

This research has been carried out in the framework of the project "Optimisation of Forming Processes MC1.03162". This project is part of the research programme of the Netherlands Institute for Metals Research (NIMR). The industrial partners co-operating in this project are gratefully acknowledged for their useful contributions to this research.

\section{REFERENCES}

1. O. Schenk, and M. Hillmann, Computers and Structures 82, 1695-1705 (2004).

2. M. Bonte, A. van den Boogaard, and J. Huétink, Submitted to: Structural and Multidisciplinary Optimization (2006).

3. K. Yang, and B. El-Haik, Design For Six Sigma; A roadmap for Product Development, McGraw-Hill, Inc., New York, USA, 2003, ISBN 0-07-141208-5.

4. M. Bonte, T. Do, L. Fourment, A. van den Boogaard, J. Huétink, and A. Habbal, "A comparison of optimisation algorithms for metal forming processes," in Proceedings of ESAFORM, Glasgow, UK, 2006, pp. 883-886.

5. R. Myers, and D. Montgomery, Response Surface Methodology: Process and Product Optimization Using Designed Experiments, John Wiley and Sons, Inc., New York, USA, 2002, $2^{\text {nd }}$ edn., ISBN 0-471-41255-4.

6. E. Veldman, Using optimisation techniques to solve a production problem, CTW.06/TM-5544, University of Twente, Enschede, The Netherlands (2006). 